\title{
E-UNITARY ALMOST FACTORIZABLE ORTHODOX SEMIGROUPS
}

\author{
MIKLÓS HARTMANN AND MÁRIA B. SZENDREI
}

\begin{abstract}
It is established that an E-unitary almost factorizable orthodox semigroup need not be isomorphic to a semidirect product of a band by a group, and a necessary and sufficient condition is given for an $E$-unitary almost factorizable orthodox semigroup to be isomorphic to such a semidirect product. Moreover, the structure of every $E$-unitary almost factorizable orthodox semigroup is described by means of bands and groups.
\end{abstract}

AMS Subject Classification: 20M19, 20M10

Key words and phrases: orthodox semigroup, E-unitary, almost factorizable, semidirect product

\section{INTRODUCTION}

Factorizable inverse monoids and almost factorizable inverse semigroups play an important role in the theory of inverse semigroups. These notions and a number of basic results are generalized in [1] for orthodox semigroups. The aim of this note is to continue these investigations. It is well known (see e.g. [3]) that an inverse semigroup is $E$-unitary and almost factorizable if and only if it is isomorphic to a semidirect product of a semilattice by a group. In this paper we give an example to show that semidirect products of bands by groups form a proper subclass of $E$-unitary almost factorizable orthodox semigroups, and provide a necessary and sufficient condition for an E-unitary almost factorizable orthodox semigroup to be isomorphic to a semidirect product of a band by a group. Moreover, we generalize the semidirect product construction, and apply it to describe the structure of E-unitary almost factorizable orthodox semigroups by means of bands and groups.

Research partially supported by the Hungarian National Foundation for Scientific Research grants no. T48809 and K60148, and by the National Development Agency grant no. TAMOP-4.2.1/B-09/1/KONV-2010-0005. 


\section{Preliminaries}

In this section we recall the notions and summarize the results of [1] needed in the paper. For the undefined notions and notation the reader is referred to [2] and [3].

The band of idempotents of an orthodox semigroup $S$ is denoted by $E(S)$, and the least inverse semigroup congruence and the least group congruence by $\gamma_{S}$ and $\sigma_{S}$, respectively. If it causes no confusion we simply write $\gamma$ and $\sigma$.

An orthodox semigroup $S$ is termed E-unitary if $E(S)$ is a unitary subset in $S$, or equivalently, if the identity class of the least group congruence of $S$, called also the kernel of this congruence, is $E(S)$. A congruence $\theta$ on an orthodox semigroup is said to be $E$-unitary if the factor semigroup $S / \theta$ is $E$-unitary.

Let $S$ be an orthodox semigroup and denote the translational hull of $S$, as usual, by $\Omega(S)$. Recall that the left translations are written as left maps and the right translations as right maps. Obviously $\Omega(S)$ is a monoid with identity $\left(\mathrm{id}_{S}, \mathrm{id}_{S}\right)$ where $\mathrm{id}_{S}$, or simply id, is the identity map of $S$. For brevity, denote the group of units of $\Omega(S)$ by $\Sigma(S)$. It is well known that $\nu_{S}: S \rightarrow \Omega(S), s \mapsto\left(\lambda_{s}, \rho_{s}\right)$ is an embedding. The orthodox semigroup $S$ is said to be almost factorizable if, for every $s \in S$, there exist $e \in E(S)$ and $(\lambda, \rho) \in \Sigma(S)$ such that $s=e \rho$. Notice that this notion is self dual, since, for every $s \in S$, there exist $e \in E(S)$ and $(\lambda, \rho) \in \Sigma(S)$ with $s=e \rho$ if and only if there exist $e^{\prime} \in E(S)$ and $\left(\lambda^{\prime}, \rho^{\prime}\right) \in \Sigma(S)$ such that $s=\lambda^{\prime} e^{\prime}$.

If $B$ is a band and $G$ is a group then we say that $G$ acts on $B$ if a homomorphism of $G$ into Aut $^{\mathrm{d}} B$, the dual of the group of automorphisms of $B$, is given. The elements of uut $^{\mathrm{d}} B$ are considered to be left maps. For a band $B$ and a group $G$ acting on $B$, we define the semidirect product of $B$ by $G$ such that its underlying set is $B \times G$ and the multiplication is defined in the following manner: for every $(a, g),(b, h) \in B \times G$,

$$
(a, g)(b, h)=\left(a \cdot{ }^{g} b, g h\right)
$$

The semigroup obtained in this way is denoted by $B \rtimes G$. Notice that each semidirect product of a band by a group is an $E$-unitary almost factorizable orthodox semigroup. The almost factorizable orthodox semigroups are characterized as follows:

Result 2.1. ([1]) An orthodox semigroup is almost factorizable if and only if it is an idempotent separating homomorphic image of a semidirect product of a band by a group. 
Let $B$ be a band and $G$ a group acting on $B$. It is well known that the second projection

$$
\pi: B \rtimes G \rightarrow G,(a, g) \mapsto g
$$

is a surjective homomorphism which induces the least group congruence on $B \rtimes G$. Since $\mathcal{D}$ is the least semilattice congruence on $B$, each automorphism respects $\mathcal{D}$, and so each automorphism $\alpha$ of $B$ determines an automorphism $\bar{\alpha}$ on the factor semilattice $B / \mathcal{D}$ by the rule $D_{a} \bar{\alpha}=D_{a \alpha}(a \in B)$. Thus the action of $G$ on $B$ determines an action of $G$ on $B / \mathcal{D}$ where ${ }^{g} D_{a}=D_{g_{a}}$ for every $a \in B$ and $g \in G$. The semidirect products $B \rtimes G$ and $(B / \mathcal{D}) \rtimes G$ defined by these actions closely relate to each other: the mapping

$$
\delta: B \rtimes G \rightarrow(B / \mathcal{D}) \rtimes G,(a, g) \mapsto\left(D_{a}, g\right)
$$

is a surjective homomorphism which induces the least inverse semigroup congruence on $B \rtimes G$. Denoting the second projection of $(B / \mathcal{D}) \rtimes G$ by $\bar{\pi}$, we cleary have $\pi=\delta \bar{\pi}$.

Let $N \triangleleft G$. Obviously, the action of $G$ on $B$ defines an action of $N$ on $B$. We say that $N$ acts identically on $B$ if ${ }^{n} a=a$ for every $a \in B$ and $n \in N$. If this is the case, then ${ }^{g} a={ }^{h} a$ for any $a \in B$ and $g, h \in G$ with $N g=N h$. Therefore an action of $G / N$ on $B$ is defined by the rule ${ }^{N g} a={ }^{g} a$, and the mapping

$$
\zeta_{N}: B \rtimes G \rightarrow B \rtimes(G / N),(a, g) \mapsto(a, N g)
$$

is a surjective homomorphism. If $N$ acts identically on $B / \mathcal{D}$ then the respective surjective homomorphism $(B / \mathcal{D}) \rtimes G \rightarrow(B / \mathcal{D}) \rtimes(G / N)$, $\left(D_{a}, g\right) \mapsto\left(D_{a}, N g\right)$ is denoted by $\bar{\zeta}_{N}$.

The proof of Result 2.1 (see [1, Theorem 5]) provides, for any almost factorizable orthodox semigroup $S$, a semidirect product $E(S) \rtimes \Sigma(S)$ of $E(S)$ by $\Sigma(S)$ such that the mapping

$$
\omega: E(S) \rtimes \Sigma(S) \rightarrow S,(e,(\lambda, \rho)) \omega=e \rho
$$

is a surjective idempotent separating homomorphism. The action

$$
\beta: \Sigma(S) \rightarrow \operatorname{Aut}^{\mathrm{d}}(E(S))
$$

of $\Sigma(S)$ on $E(S)$ involved in the definition of this semidirect product is defined, for any $e \in E(S)$ and any $(\lambda, \rho) \in \Sigma(S)$, by the rule

$$
{ }^{(\lambda, \rho)} e=\lambda e \rho^{-1} \text {. }
$$

Recall that, in a regular semigroup, for any element $a$, and for any left translation $\lambda$ and any right translation $\rho$, we have $(\lambda a) \rho=\lambda(a \rho)$, therefore parentheses can be omitted. 
It is also shown in [1, Section 5] that any inverse semigroup homomorphic image of a semidirect product of a band by a group is necessarily an almost factorizable inverse semigroup. In particular, by Result 2.1, the greatest inverse semigroup homomorphic image of any almost factorizable orthodox semigroup is almost factorizable. Therefore the analogues of (2.4) and (2.6) can be introduced for $S / \gamma$. We have $E(S / \gamma)=E(S) / \mathcal{D}$ on which $\Sigma(S / \gamma)$ acts by the rule ${ }^{(\bar{\lambda}, \bar{\rho})} D_{e}=\bar{\lambda} D_{e} \bar{\rho}^{-1}$. This determines a semidirect product $(E(S) / \mathcal{D}) \rtimes \Sigma(S / \gamma)$ and a surjective homomorphism

$$
\bar{\omega}:(E(S) / \mathcal{D}) \rtimes \Sigma(S / \gamma) \rightarrow S / \gamma,\left(D_{e},(\bar{\lambda}, \bar{\rho})\right) \bar{\omega}=D_{e} \bar{\rho} .
$$

The following observation allows us to define a mapping from $\Sigma(S)$ to $\Sigma(S / \gamma)$ in a natural way for any orthodox semigroup $S$.

Lemma 2.2. Let $S$ be an orthodox semigroup and let $(\lambda, \rho) \in \Sigma(S)$. Define

$$
\lambda_{\gamma}: S / \gamma \rightarrow S / \gamma, \lambda_{\gamma}(s \gamma)=(\lambda s) \gamma
$$

and

$$
\rho_{\gamma}: S / \gamma \rightarrow S / \gamma,(s \gamma) \rho_{\gamma}=(s \rho) \gamma
$$

Then $\left(\lambda_{\gamma}, \rho_{\gamma}\right) \in \Sigma(S / \gamma)$.

Proof. Let $(\lambda, \rho) \in \Sigma(S)$. If $s \in S$ and $x \in V(s)$ then $\lambda^{-1} x \in V(s \rho)$. For, we have

$$
\begin{aligned}
s \rho \cdot \lambda^{-1} x \cdot s \rho & =\left(s \rho \cdot \lambda^{-1} x \cdot s\right) \rho=\left(s \cdot \lambda\left(\lambda^{-1} x\right) \cdot s\right) \rho \\
& =(s x s) \rho=s \rho
\end{aligned}
$$

and

$$
\begin{aligned}
\lambda^{-1} x \cdot s \rho \cdot \lambda^{-1} x & =\lambda^{-1}\left(x \cdot s \rho \cdot \lambda^{-1} x\right)=\lambda^{-1}\left(x \cdot s \cdot \lambda\left(\lambda^{-1} x\right)\right) \\
& =\lambda^{-1}(x s x)=\lambda^{-1} x .
\end{aligned}
$$

Therefore if $s \gamma t$ in $S$ then $s \rho \gamma t \rho$ also holds. This ensures that $\rho_{\gamma}$ is well defined. It is routine to check that $\rho_{\gamma}$ is, indeed, a right translation of $S / \gamma$. Moreover, $\rho_{\gamma}\left(\rho^{-1}\right)_{\gamma}=\left(\rho^{-1}\right)_{\gamma} \rho_{\gamma}=\mathrm{id}_{S / \gamma}$, and so $\rho_{\gamma}$ is bijective and $\left(\rho_{\gamma}\right)^{-1}=\left(\rho^{-1}\right)_{\gamma}$. From now on, for the latter right translation we simply write $\rho_{\gamma}^{-1}$. Similarly, we obtain that $\lambda_{\gamma}$ is also a well-defined bijective left translation of $S / \gamma$, and we have $\left(\lambda_{\gamma}\right)^{-1}=\left(\lambda^{-1}\right)_{\gamma}$. The latter left translation is denoted briefly by $\lambda_{\gamma}^{-1}$. Finally, it is also immediate by definition that $\lambda_{\gamma}$ and $\rho_{\gamma}$ are linked, and so $\left(\lambda_{\gamma}, \rho_{\gamma}\right) \in \Sigma(S / \gamma)$.

Let us define, for any orthodox semigroup $S$, the mapping

$$
\chi: \Sigma(S) \rightarrow \Sigma(S / \gamma),(\lambda, \rho) \chi=\left(\lambda_{\gamma}, \rho_{\gamma}\right) .
$$

Obviously, $\chi$ is a group homomorphism. 
By definitions and by the proof of the previous lemma, the actions of $\Sigma(S)$ on $E(S)$ and of $\Sigma(S / \gamma)$ on $E(S) / \mathcal{D}$ defined above are closely related: we have

$$
\begin{aligned}
D_{(\lambda, \rho) e} & =\left({ }^{(\lambda, \rho)} e\right) \gamma=\left(\lambda e \rho^{-1}\right) \gamma \\
& =\lambda_{\gamma}(e \gamma) \rho_{\gamma}^{-1}={ }^{\left(\lambda_{\gamma}, \rho_{\gamma}\right)} D_{e}
\end{aligned}
$$

for every $e \in E(S)$ and $(\lambda, \rho) \in \Sigma(S)$. This implies that the mapping

$$
\begin{aligned}
\vartheta: E(S) \rtimes \Sigma(S) \rightarrow(E(S) / \mathcal{D}) \rtimes \Sigma(S / \gamma), \\
(e,(\lambda, \rho)) \vartheta=\left(D_{e},\left(\lambda_{\gamma}, \rho_{\gamma}\right)\right)
\end{aligned}
$$

is a homomorphism. Moreover, we have

$$
\omega \gamma^{\natural}=\vartheta \bar{\omega} \text {. }
$$

Note that if the homomorphism $\chi$ is surjective, and we identify $\Sigma(S / \gamma)$ with the factor group $\Sigma(S) / \operatorname{Ker} \chi$ then we have $\vartheta=\delta \bar{\zeta}_{\text {Ker } \chi}$ where the homomorphisms $\delta: E(S) \rtimes \Sigma(S) \rightarrow(E(S) / \mathcal{D}) \rtimes \Sigma(S)$ and $\bar{\zeta}_{\text {Ker } \chi}:(E(S) / \mathcal{D}) \rtimes \Sigma(S) \rightarrow(E(S) / \mathcal{D}) \rtimes(\Sigma(S) /$ Ker $\chi)$ are defined as above.

\section{The structure of E-Unitary almost FaCtorizable ORTHODOX SEMIGROUPS}

In this section we characterize the idempotent separating $E$-unitary congruences on a semidirect product of a band by a group, and describe the structure of any $E$-unitary almost factorizable orthodox semigroup as a Schreier-type extension of a band by a group.

Proposition 3.1. Let $B$ be a band and $G$ be a group acting on $B$. Let $N \triangleleft G$ such that the following condition is satisfied for every $a, b \in B$ and $n \in N$ :

(C) ${ }^{n}(a b) \cdot b \mathcal{L} a b \mathcal{R} a \cdot{ }^{n}(a b)$.

Define a relation on the semidirect product $B \rtimes G$ in the way that, for every $(a, g),(b, h) \in B \rtimes G$, let

$$
(a, g) \kappa(b, h) \quad \Leftrightarrow \quad N g=N h, a \mathcal{R} b \text { and } g^{-1} a \mathcal{L}^{h^{-1}} b .
$$

Then

(1) $\kappa$ is an idempotent separating E-unitary congruence on $B \rtimes G$,

(2) $\phi:(B \rtimes G) / \kappa \rightarrow G / N,(a, g) \kappa \mapsto N g$ is a surjective and idempotent pure homomorphism, and so the greatest group homomorphic image of $(B \rtimes G) / \kappa$ is $G / N$.

Conversely, each idempotent separating E-unitary congruence $\kappa$ on the semidirect product $B \rtimes G$ is of this form for a unique normal subgroup $N$ in $G$. 
Proof. Let $\kappa$ be an idempotent separating $E$-unitary congruence on $B \rtimes G$, and put $S=(B \rtimes G) / \kappa$. Denote the least group congruence on $S$ by $\sigma$. Since the congruence induced by the second projection $\pi: B \rtimes G \rightarrow G$ is the least group congruence on $B \rtimes G$, there is a unique homomorphism $\tau: G \rightarrow S / \sigma$ with $\pi \tau=\kappa^{\natural} \sigma^{\natural}$, see Diagram 1 .

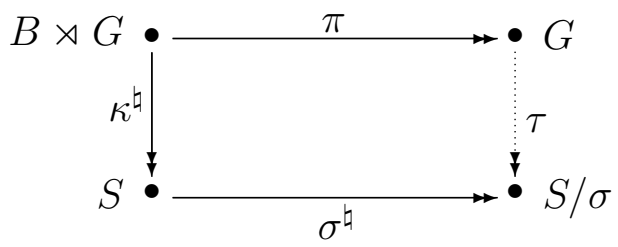

Diagram 1

Denote the kernel of the group homomorphism $\tau$ by $N$. Assume that $(a, g),(b, h) \in B \rtimes G$ with $(a, g) \kappa(b, h)$. Observe that in this case, we have $N g=N h$. Since $\kappa$ is idempotent separating, we also have that $(a, g) \mathcal{R}(b, h)$ and $(a, g) \mathcal{L}(b, h)$. This implies that

$$
a \mathcal{R} b \quad \text { and } \quad g^{-1} a \mathcal{L}^{h^{-1}} b .
$$

In particular, we infer for every $a, b \in B$ and $n \in N$ that

$$
(a, n) \kappa(b, 1) \quad \text { if and only if } \quad a \mathcal{R} b \text { and } \quad n^{-1} a \mathcal{L} b .
$$

Since $S$ is $E$-unitary, we have $(a, n) \kappa \in E(S)$. Hence, for every $a \in B$ and $n \in N$, there exists a unique $b \in B$ such that $(a, n) \kappa(b, 1)$. By (3.2) this implies that $a \mathcal{D}^{n^{-1}} a$ and $b=a \cdot{ }^{n^{-1}} a$. Moreover, the property that $a \mathcal{D}^{n} a$ for any $a \in B$ and $n \in N$ ensures that, for every $b \in B$ and $n \in N$, there exists a unique $a \in B$ such that $b \mathcal{R} a \mathcal{L}^{n} b$, and so $(a, n) \kappa(b, 1)$ follows by $(3.2)$. In this case, we have $a=b \cdot{ }^{n} b$.

Now let $a, b \in B$ and $n, q \in N$. By the previous observation, we have $(a, 1) \kappa\left(a \cdot{ }^{n} a, n\right)$ and $(b, 1) \kappa\left(b \cdot{ }^{q} b, q\right)$, and since $\kappa$ is a congruence, we see that

$$
(a b, 1)=(a, 1)(b, 1) \kappa\left(a \cdot{ }^{n} a, n\right)\left(b \cdot{ }^{q} b, q\right)=\left(a \cdot{ }^{n} a \cdot{ }^{n} b \cdot{ }^{n q} b, n q\right) .
$$

By applying property (3.1) and the relations

$$
a \mathcal{D}^{n} a \mathcal{D}^{q^{-1}} a \mathcal{D}^{(n q)^{-1}} a \text { and } b \mathcal{D}^{n} b \mathcal{D}^{q^{-1}} b \mathcal{D}^{n q} b,
$$

we deduce that $a b \mathcal{R} a \cdot{ }^{n}(a b)$ and $a b \mathcal{L}^{(n q)^{-1}}\left({ }^{n}(a b) \cdot{ }^{n q} b\right)=q^{-1}(a b) \cdot b$. Since $n, q \in N$ are arbitrary, thus we have proved that each idempotent separating $E$-unitary congruence on $B \rtimes G$ is of the form stated in the proposition.

In order to show the converse part, we have to verify that the relation $\kappa$ defined in the proposition is, indeed, an idempotent separating $E$ unitary congruence on $B \rtimes G$. It is immediate that $\kappa$ is an equivalence. 
Let $(a, g),(b, h),(c, k) \in B \rtimes G$ such that $(a, g) \kappa(b, h)$, that is, $N g=$ $N h$ and (3.1) holds. In order to check left compatibility of $\kappa$, we need to show that the elements $(c, k)(a, g)=\left(c \cdot{ }^{k} a, k g\right)$ and $(c, k)(b, h)=$ $\left(c \cdot{ }^{k} b, k h\right)$ are $\kappa$-related. Here $N g=N h$ and (3.1) imply $N k g=k N g=$ $k N h=N k h$ and $c \cdot{ }^{k} a \mathcal{R} c \cdot{ }^{k} b$, respectively. Finally, we check that the

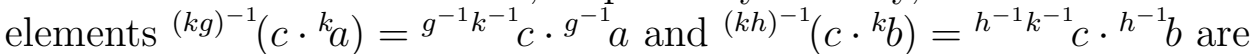
$\mathcal{L}$-related. By applying condition (C) with the element $h^{-1} g \in N$, we

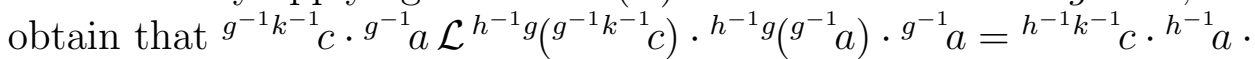
$g^{-1} a=h^{-1} k^{-1} c \cdot h^{-1}\left(a \cdot h g^{-1} a\right)$. Here we have $h g^{-1} \in N$ and, again by (3.1), we have $a \mathcal{R} b \mathcal{L}^{h g^{-1}} a$ whence $b=a \cdot{ }^{h g^{-1}} a$. Thus we see that $\kappa$ is left compatible. Right compatibility follows dually, and so $\kappa$ is, indeed, a congruence.

It is obvious by the definition of $\kappa$ that it is idempotent separating. Moreover, the mapping $\phi:(B \rtimes G) / \kappa \rightarrow G / N,(a, g) \kappa \mapsto N g$ is a homomorphism of $(B \rtimes G) / \kappa$ onto $G / N$. We intend to show that the kernel $K_{\phi}$ of the group congruence of $(B \rtimes G) / \kappa$ induced by $\phi$, that is, $K_{\phi}=\{(a, n) \kappa: a \in B, n \in N\}$ is just $E((B \rtimes G) / \kappa)$. This directly implies that $\phi$ is idempotent pure, $(B \rtimes G) / \kappa$ is $E$-unitary, and its greatest group homomorphic image is $G / N$. In order to see the statement formulated, first notice that property $(\mathrm{C})$ implies that, for every $a \in B$ and $n \in N$, we have

$$
a \mathcal{D}^{n} a .
$$

For, by (C), we have $a=a \cdot a \mathcal{R} a \cdot{ }^{n}(a \cdot a)=a \cdot{ }^{n} a$ for any $a \in B$ and $n \in N$. Therefore also ${ }^{n} a \mathcal{R}^{n} a \cdot{ }^{n-1}\left({ }^{n} a\right)={ }^{n} a \cdot a$ hold whence $a \mathcal{D}^{n} a$ follows. This observation immediately implies that, for any $a \in B$ and $n \in N$, we have $a \mathcal{R} a \cdot{ }^{n^{-1}} a$ and ${ }^{n^{-1}} a \mathcal{L} a \cdot{ }^{-1} a$. Thus each element $(a, n) \in B \rtimes G$ with $a \in B, n \in N$ is $\kappa$-related to the idempotent $\left(a \cdot n^{-1} a, 1\right)$, and so $(a, n) \kappa$ is idempotent. Hence $K_{\phi}=E((B \rtimes G) / \kappa)$ is verified.

Finally, notice that the kernel of the unique group homomorphism $\tau: G \rightarrow(B \rtimes G) / \kappa) / \sigma$ which satisfies $\pi \tau=\kappa^{\natural} \sigma^{\natural}$ is just $N$ by the property verified in the previous paragraph. Therefore $N$ is, indeed, uniquely determined.

By Result 2.1, we can formulate the following corollary.

Corollary 3.2. Each E-unitary almost factorizable orthodox semigroup is isomorphic to a factor semigroup of a semidirect product $B \rtimes G$ of a band by a group modulo a congruence $\kappa$ defined, as in Proposition 3.1, by means of a normal subgroup $N$ of $G$ satisfying condition (C) in Proposition 3.1. 
The use of a factor semigroup can be avoided from the construction by representing each class with an element. This leads to a description of the structure of $E$-unitary almost factorizable orthodox semigroups. Before continuing in this direction, we formulate another corollary to Proposition 3.1 for later use.

We have noticed in the proof of Proposition 3.1 that property (C) implies (3.3) for every $a \in B$ and $n \in N$, that is, that $N$ acts identically on $B / \mathcal{D}$. Conversely, if $B$ is a normal band then this property implies (C). By applying that $\mathcal{R}$ is the equality relation for any left normal band $B$ and each of $\mathcal{R}, \mathcal{L}, \mathcal{D}$ is the equality relation for any semilattice, we can simplify Proposition 3.1 for left normal bands and, in particular, for semilattices, as follows.

Corollary 3.3. Let $B$ be a left normal band [semilattice] and $G$ be a group acting on $B$. Let $N \triangleleft G$ which acts identically on $B / \mathcal{D}[B]$. Define a relation on the semidirect product $B \rtimes G$ in the way that, for every $(a, g),(b, h) \in B \rtimes G$, let

$$
(a, g) \kappa(b, h) \quad \Leftrightarrow \quad N g=N h \text { and } a=b .
$$

Then

(1) $\kappa$ is an idempotent separating E-unitary congruence on $B \rtimes G$,

(2) $\phi:(B \rtimes G) / \kappa \rightarrow G / N,(a, g) \kappa \mapsto N g$ is a surjective and idempotent pure homomorphism, and so the greatest group homomorphic image of $(B \rtimes G) / \kappa$ is $G / N$.

Conversely, each idempotent separating E-unitary congruence $\kappa$ on the semidirect product $B \rtimes G$ is of this form for a unique normal subgroup $N$ in $G$.

Returning to the study of the structure of $E$-unitary almost factorizable orthodox semigroups, now we introduce a Schreier-type generalization of a semidirect product of a band by a group, and apply this construction to describe the structure of these semigroups by means of bands and groups.

Construction 3.4. Let $B$ be a band and $H$ a group. Consider a mapping $\xi: H \rightarrow$ Aut $^{\mathrm{d}} B, h \mapsto \xi_{h}$ such that the following conditions are satisfied by $\xi$ and by the mapping

$$
\eta: H \times H \rightarrow \operatorname{Aut}^{\mathrm{d}} B,(h, k) \mapsto \eta_{h, k}=\xi_{h k} \xi_{k}^{-1} \xi_{h}^{-1}:
$$

(Q0) $\xi_{1}=\mathrm{id}_{B}$,

(Q1) $a \mathcal{D} \eta_{h, k} a$ for every $h, k \in H$ and $a \in B$,

(Q2) $\eta_{h, k}(a b) \cdot b \mathcal{L} a b \mathcal{R} a \cdot \eta_{h, k}(a b)$ for every $h, k \in H$ and $a, b \in B$. 
Define a multiplication on the set $B \times H$ in the following manner: for every $(a, h),(b, k) \in B \times H$, let

$$
(a, h)(b, k)=\left(a \cdot \xi_{h} b \cdot \eta_{h, k}\left(a \cdot \xi_{h} b\right), h k\right) .
$$

Denote the structure obtained by $Q(B, H ; \xi)$, and refer to it as a $Q$ product of $B$ by $H$.

Notice that this construction is a generalization of a semidirect product of a band by a group. Indeed, $\xi$ is a homomorphism if and only if $\eta$ is trivial, that is, $\eta_{h, k}=\mathrm{id}_{B}$ for every $h, k \in H$. In this case (Q0)-(Q2) trivially hold, and so $Q(B, H ; \xi)=B \rtimes H$ where the action of $H$ on $B$ involved in the semidirect product is given by $\xi$.

Theorem 3.5. Let $B$ be $a$ band and $H$ a group. Let $\xi: H \rightarrow$ Aut $^{\mathrm{d}} B$ be a mapping such that conditions (Q0)-(Q2) are satisfied. Then the $Q$-product $Q(B, H ; \xi)$ of $B$ by $H$ is an E-unitary almost factorizable orthodox semigroup. Conversely, each E-unitary almost factorizable orthodox semigroup is isomorphic to a $Q$-product of a band by a group.

Proof. Let $S=Q(B, H ; \xi)$ be as defined in Construction 3.4. First we establish several consequences of conditions (Q0)-(Q2). By the definition of $\eta$ it is straightforward to see that, for any $h, k \in H$, we have

(Q3) $\eta_{1, h}=\eta_{h, 1}=\mathrm{id}_{B}$,

(Q4) $\xi_{h k}=\eta_{h, k} \xi_{h} \xi_{k}$.

Moreover, for any $h, k, l \in H$, we have

(Q5) $\eta_{h k, l} \eta_{h, k} \xi_{h}=\eta_{h, k l} \xi_{h} \eta_{k, l}$.

For, we obtain from (Q4) that

$$
\xi_{h k l}=\eta_{h k, l} \xi_{h k} \xi_{l}=\eta_{h k, l} \eta_{h, k} \xi_{h} \xi_{k} \xi_{l}
$$

and

$$
\xi_{h k l}=\eta_{h, k l} \xi_{h} \xi_{k l}=\eta_{h, k l} \xi_{h} \eta_{k, l} \xi_{k} \xi_{l},
$$

whence (Q5) follows.

Denote by $\langle\eta\rangle$ the subgroup of Aut ${ }^{\mathrm{d}} B$ generated by the subset $\left\{\eta_{h, k}\right.$ : $h, k \in H\}$. The following relations generalize (Q1) and (Q2): we have

(Q6) $a \mathcal{D} \alpha a$ for every $\alpha \in\langle\eta\rangle$ and $a \in B$,

(Q7) $\alpha(a b) \cdot b \mathcal{L} a b \mathcal{R} a \cdot \alpha(a b)$ for every $\alpha \in\langle\eta\rangle$ and $a, b \in B$.

Consider the following subsets in $\mathrm{Aut}^{\mathrm{d}} B$ :

$$
\begin{aligned}
& A_{\mathcal{D}}=\left\{\alpha \in \text { Aut }^{\mathrm{d}} B: a \mathcal{D} \alpha a \text { for every } a \in B\right\}, \\
& A_{\mathcal{L}}=\left\{\alpha \in \operatorname{Aut}^{\mathrm{d}} B: a b \mathcal{L} \alpha(a b) \cdot b \text { for every } a, b \in B\right\}, \\
& A_{\mathcal{R}}=\left\{\alpha \in \operatorname{Aut}^{\mathrm{d}} B: a b \mathcal{R} a \cdot \alpha(a b) \text { for every } a, b \in B\right\} .
\end{aligned}
$$


Since $\eta_{h, k} \in A_{\mathcal{D}}, A_{\mathcal{L}}, A_{\mathcal{R}}$ for every $h, k \in H$ by (Q1) and (Q2), properties (Q6) and (Q7) follow if we show that $A_{\mathcal{D}}, A_{\mathcal{L}}, A_{\mathcal{R}}$ are subgroups in Aut ${ }^{\mathrm{d}} B$. Clearly, $\operatorname{id}_{B} \in A_{\mathcal{D}}, A_{\mathcal{R}}$. If $\alpha, \alpha^{\prime} \in A_{\mathcal{D}}$ then, for any $a \in B$, we deduce by (Q1) that $\alpha^{-1} a \mathcal{D} \alpha\left(\alpha^{-1} a\right) \mathcal{D} a$ and $\left(\alpha^{\prime} \alpha\right) a \mathcal{D} \alpha^{\prime}(\alpha a) \mathcal{D} \alpha a \mathcal{D} a$. Thus $A_{\mathcal{D}}$ is, indeed, a subgroup, and so (Q6) is verified. Now let $\alpha, \alpha^{\prime} \in$ $A_{\mathcal{R}}$ and $a, b \in B$. Then $a b \mathcal{R} a \cdot \alpha(a b)$ implies $\alpha^{-1}(a b) \mathcal{R} \alpha^{-1}(a \cdot \alpha(a b))=$ $\alpha^{-1} a \cdot a b$. Hence $a \cdot \alpha^{-1}(a b) \mathcal{R} a \cdot \alpha^{-1} a \cdot a b=a b$ follows where, in the last equality, we apply (Q6). Similarly, the relation $a b \mathcal{R} a \cdot \alpha(a b)$ implies $\alpha^{\prime}(a b) \mathcal{R} \alpha^{\prime} a \cdot\left(\alpha^{\prime} \alpha\right)(a b)$, whence we deduce

$$
a \cdot \alpha^{\prime}(a b) \mathcal{R} a \cdot \alpha^{\prime} a \cdot\left(\alpha^{\prime} \alpha\right)(a b)=a \cdot\left(\alpha^{\prime} \alpha\right)(a b) .
$$

In the last step we utilize that $a, \alpha^{\prime} a$ and $\left(\alpha^{\prime} \alpha\right) a$ are $\mathcal{D}$-related elements in $B$ by (Q6). Since $a b \mathcal{R} a \cdot \alpha^{\prime}(a b)$, we obtain from the former relation that $a b \mathcal{R} a \cdot\left(\alpha^{\prime} \alpha\right)(a b)$. Therefore we see that $A_{\mathcal{R}}$ is a subgroup, and a dual argument shows that the same holds for $A_{\mathcal{L}}$. This completes the proof of (Q7).

In order to check that $S$ is a semigroup, consider arbitrary elements $(a, h),(b, k),(c, l) \in S$, and compute the products $((a, h)(b, k))(c, l)$ and $(a, h)((b, k)(c, l))$. The second components are clearly equal, the first components are

$$
x=a \cdot \xi_{h} b \cdot \eta_{h, k}\left(a \cdot \xi_{h} b\right) \cdot \xi_{h k} c \cdot \eta_{h k, l}\left(a \cdot \xi_{h} b \cdot \eta_{h, k}\left(a \cdot \xi_{h} b\right) \cdot \xi_{h k} c\right)
$$

and

$$
y=a \cdot \xi_{h}\left(b \cdot \xi_{k} c \cdot \eta_{k, l}\left(b \cdot \xi_{k} c\right)\right) \cdot \eta_{h, k l}\left(a \cdot \xi_{h}\left(b \cdot \xi_{k} c \cdot \eta_{k, l}\left(b \cdot \xi_{k} c\right)\right)\right),
$$

respectively. By (Q1), here we have

$$
x \mathcal{R} a \cdot \xi_{h} b \cdot \eta_{h, k}\left(a \cdot \xi_{h} b\right) \cdot \xi_{h k} c
$$

and

$$
y \mathcal{R} a \cdot \xi_{h} b \cdot \xi_{h} \xi_{k} c .
$$

By applying conditions (Q2) and (Q4), hence we see that

$$
\begin{aligned}
& \begin{array}{lll}
x & \mathcal{R} & a \cdot \xi_{h} b \cdot \eta_{h, k}\left(\left(a \cdot \xi_{h} b\right) \cdot \eta_{h, k}^{-1} \xi_{h k} c\right)
\end{array} \\
& \mathcal{R} a \cdot \xi_{h} b \cdot \eta_{h, k}^{-1} \xi_{h k} c=a \cdot \xi_{h} b \cdot \xi_{h} \xi_{k} c \mathcal{R} y .
\end{aligned}
$$

Similarly, we obtain from (3.4) and (3.5) that

$$
x \mathcal{L} \eta_{h k, l}\left(\eta_{h, k}\left(a \cdot \xi_{h} b\right) \cdot \xi_{h k} c\right)
$$

and

$$
y \mathcal{L} \eta_{h, k l} a \cdot \eta_{h, k l} \xi_{h}\left(b \cdot \xi_{k} c\right) \cdot \eta_{h, k l} \xi_{h} \eta_{k, l}\left(b \cdot \xi_{k} c\right)
$$


By conditions (Q5), (Q7) and (Q4), we deduce that

$$
\begin{aligned}
& y \quad \mathcal{L} \quad \eta_{h, k l} \eta_{h, k}^{-1} \eta_{h k, l}^{-1}\left(\eta_{h k, l} \eta_{h, k} a \cdot \eta_{h k, l} \eta_{h, k} \xi_{h}\left(b \cdot \xi_{k} c\right)\right) \cdot \eta_{h k, l} \eta_{h, k} \xi_{h}\left(b \cdot \xi_{k} c\right) \\
& \mathcal{L} \quad \eta_{h k, l} \eta_{h, k} a \cdot \eta_{h k, l} \eta_{h, k} \xi_{h}\left(b \cdot \xi_{k} c\right) \\
& =\eta_{h k, l} \eta_{h, k}\left(a \cdot \xi_{h} b\right) \cdot \eta_{h k, l} \eta_{h, k} \xi_{h} \xi_{k} c \\
& =\eta_{h k, l} \eta_{h, k}\left(a \cdot \xi_{h} b\right) \cdot \eta_{h k, l} \xi_{h k} c \quad \mathcal{L} \quad x .
\end{aligned}
$$

Thus $x=y$, and we have verified that $S$ is a semigroup.

Regularity of $S$ is routine to show by computing that, for every $(a, h) \in S$, we have $(a, h)\left(\xi_{h}^{-1} a, h^{-1}\right)(a, h)=(a, h)$. Here properties (Q1), (Q0) and (Q3) are applied, and the details are left to the reader. Conditions (Q0) and (Q3) also imply that $E(S)=\{(a, 1) \in S: a \in B\}$, and that $E(S)$ is closed under multiplication. Moreover, the second projection of $S$ is a surjective homomorphism to the group $H$, and the kernel of the group congruence induced by it is just $E(S)$. This implies that $S$ is an $E$-unitary orthodox semigroup.

Now we turn to proving that $S$ is almost factorizable. For any $u \in H$, let us define a right map $\rho_{u}: S \rightarrow S$ and a left map $\lambda_{u}: S \rightarrow S$ as follows: for every $(a, h) \in S$, let

$$
(a, h) \rho_{u}=\left(a \cdot \eta_{h, u} a, h u\right)
$$

and

$$
\lambda_{u}(a, h)=\left(\xi_{u} a \cdot \eta_{u, h} \xi_{u} a, u h\right) .
$$

We show that $\rho_{u}$ is a right translation of $S$. If $(a, h),(b, k) \in S$ then the second components of $(b, k)\left((a, h) \rho_{u}\right)$ and $((b, k)(a, h)) \rho_{u}$ clearly coincide, so it suffices to verify the equality of the first components

$$
x=b \cdot \xi_{k}\left(a \cdot \eta_{h, u} a\right) \cdot \eta_{k, h u}\left(b \cdot \xi_{k}\left(a \cdot \eta_{h, u} a\right)\right)
$$

and

$$
y=b \cdot \xi_{k} a \cdot \eta_{k, h}\left(b \cdot \xi_{k} a\right) \cdot \eta_{k h, u}\left(b \cdot \xi_{k} a \cdot \eta_{k, h}\left(b \cdot \xi_{k} a\right)\right) .
$$

Observe that, by (Q1), the relation $x \mathcal{R} b \cdot \xi_{k} a \mathcal{R} y$ clearly holds. Furthermore, we have

$$
x \mathcal{L} \eta_{k, h u}\left(b \cdot \xi_{k}\left(a \cdot \eta_{h, u} a\right)\right) \quad \text { and } \quad y \mathcal{L} \eta_{k h, u} \eta_{k, h}\left(b \cdot \xi_{k} a\right) .
$$

Applying (Q5) and (Q7), we obtain that

$$
\begin{array}{rl}
x & \mathcal{L} \\
= & \eta_{k, h u}\left(b \cdot \xi_{k} a\right) \cdot \eta_{k, h u} \xi_{k} \eta_{h, u} a \\
= & \eta_{k, h u}\left(b \cdot \xi_{k} a\right) \cdot \eta_{k h, u} \eta_{k, h} \xi_{k} a \\
= & \eta_{k, h u} \eta_{k, h}^{-1} \eta_{k h, u}^{-1}\left(\eta_{k h, u} \eta_{k, h} b \cdot \eta_{k h, u} \eta_{k, h} \xi_{k} a\right) \cdot \eta_{k h, u} \eta_{k, h} \xi_{k} a \\
\mathcal{L} & \eta_{k h, u} \eta_{k, h}\left(b \cdot \xi_{k} a\right) \mathcal{L} y .
\end{array}
$$


Thus $x=y$ follows, and so $\rho_{u}$ is proved to be a right translation. It is injective since, for every $(a, h),(b, k) \in S$ with $(a, h) \rho_{u}=(b, k) \rho_{u}$, we see that $h=k$ and $a \cdot \eta_{h, u} a=b \cdot \eta_{h, u} b$. Therefore (Q1) implies $a \mathcal{R} b$ and $\eta_{h, u} a \mathcal{L} \eta_{h, u} a$ whence $a=b$ follows. The right translation $\rho_{u}$ is also surjective since, again by (Q1),

$$
\left(a \cdot \eta_{h u^{-1}, u}^{-1} a, h u^{-1}\right) \rho_{u}=\left(a \cdot \eta_{h u^{-1}, u}^{-1} a \cdot \eta_{h u^{-1}, u}\left(a \cdot \eta_{h u^{-1}, u}^{-1} a\right), h\right)=(a, h) .
$$

Notice that, in particular, this implies by (Q3) that any element $(a, h) \in$ $S$ is of the form $(a, 1) \rho_{u}$ where $(a, 1) \in E(S)$ and $u=h$. Dually, one can verify that $\lambda_{u}$ is a bijective left translation for any $u \in H$. The fact that the pair $\left(\lambda_{u}, \rho_{u}\right)$ is linked is checked by carrying over similar calculations on $(a, h) \rho_{u} \cdot(b, k)$ and $(a, h) \cdot \lambda_{u}(b, k)$ as above. Thus $\left(\lambda_{u}, \rho_{u}\right) \in \Sigma(S)$. This completes the proof of the fact that $S=Q(B, H ; \xi)$ is an $E$ unitary almost factorizable orthodox semigroup.

Conversely, let $S$ be an $E$-unitary almost factorizable orthodox semigroup. Then, by Proposition 3.1, $S$ is isomorphic to a factor semigroup $(B \rtimes G) / \kappa$. For simplicity, assume that $S=(B \rtimes G) / \kappa$. Put $H=G / N$, and choose and fix, for every $h \in H$, an element $r_{h} \in G$ from the coset $h$, that is, an element $r_{h} \in G$ with $N r_{h}=h$. In particular, let $r_{1}=1$. Since $N r_{h k}=N r_{h} r_{k}$ for any $h, k \in H$, there is a unique $n \in N$ with $r_{h k}=n r_{h} r_{k}$. Denote this element $n$ by $n_{h, k}$. Denote the automorphisms of $B$ assigned by the action of $G$ on $B$ to $r_{h}$ and $n_{h, k}(h, k \in H)$ by $\xi_{h}$ and $\eta_{h, k}$, respectively. By the choices of $r_{h}$ and $n_{h, k}(h, k \in H)$ we immediately see that $\xi$ satisfies condition (Q0), and that $\eta_{h, k}$ is just $\xi_{h k} \xi_{k}^{-1} \xi_{h}^{-1}$. Property (C) in Proposition 3.1 and relation (3.3) imply that conditions (Q1) and (Q2) are also fulfilled. Thus the semigroup $Q(B, H ; \xi)$ is defined.

Observe by the definition of $\kappa$ that, for any $(a, g) \in B \rtimes G$, there exist uniquely determined $h \in H$ and $b \in B$ such that $(a, g) \kappa\left(b, r_{h}\right)$, namely, $h=N g$ and $b=a \cdot r_{h} g^{-1} a$. In deducing the latter equality we apply that $r_{h} g^{-1} \in N$ and that (3.3) is valid. Define a mapping $\psi: S \rightarrow Q(B, H ; \xi)$ by the rule $((a, g) \kappa) \psi=\left(a \cdot{ }^{r_{N g} g^{-1}} a, N g\right)$. This mapping is obvouisly surjective because $\left(\left(a, r_{h}\right) \kappa\right) \psi=(a, h)$ for every $(a, h) \in Q(B, H ; \xi)$. It is also injective since if $((a, g) \kappa) \psi=((b, q) \kappa) \psi$ then $N g=N q$ in $H$ and, putting $h=N g=N q$, we have $a \cdot{ }^{r_{h}} g^{-1} a=$ $b \cdot r_{h} q^{-1} b$. Applying (3.3), we obtain that $a \mathcal{R} b$ and $g^{-1} a \mathcal{L}^{q^{-1}} b$ which implies $(a, g) \kappa(b, q)$ by definition. Finally, we verify that $\psi$ is a homomorphism. By definition, we have

$$
\begin{aligned}
\left(\left(a, r_{h}\right) \kappa\right) \psi \cdot\left(\left(b, r_{k}\right) \kappa\right) \psi & =(a, h)(b, k) \\
& =\left(a \cdot \xi_{h} b \cdot \eta_{h, k}\left(a \cdot \xi_{h} b\right), h k\right)
\end{aligned}
$$


and

$$
\begin{aligned}
\left(\left(\left(a, r_{h}\right) \kappa\right)\left(\left(b, r_{k}\right) \kappa\right)\right) \psi & =\left(\left(a \cdot{ }^{r_{h}} b, r_{h} r_{k}\right) \kappa\right) \psi \\
& =\left(\left(a \cdot{ }^{r} b \cdot r_{h k}\left(r_{h} r_{k}\right)^{-1}\left(a \cdot{ }^{r_{h}} b\right), r_{h k}\right) \kappa\right) \psi \\
& =\left(\left(a \cdot{ }^{r} b \cdot{ }^{n_{h, k}}\left(a \cdot{ }^{r} b\right), r_{h k}\right) \kappa\right) \psi \\
& =\left(a \cdot \xi_{h} b \cdot \eta_{h, k}\left(a \cdot \xi_{h} b\right), h k\right) .
\end{aligned}
$$

Thus $\psi$ is an isomorphism, and so $S$ is isomorphic to $Q(B, H ; \xi)$.

Combining Result 2.1, Theorem 3.5, its proof and the observations in Section 2, we can describe the structure of an E-unitary almost factorizable orthodox semigroup by means of internal parameters.

We have already mentioned that if $S$ is an almost factorizable orthodox semigroup then $S / \gamma$ is an almost factorizable inverse semigroup. Since $\gamma \subseteq \sigma$, it is clear that if $S$ is $E$-unitary then so is $S / \gamma$. Therefore if $S$ is an $E$-unitary almost factorizable orthodox semigroup then $S / \gamma$ is an $E$-unitary almost factorizable inverse semigroup. It is well known that in this case, $S / \gamma$ is isomorphic to a semidirect product of a semilattice by a group. More precisely, one can deduce by combining [3, Theorem 7.1.8], [4, Theorem V.2.8] and [1, Lemma 3] that the homomorphism $\bar{\omega}$ defined in (2.7) is, in fact, an isomorphism. Therefore (2.10) implies that the homomorphism $\vartheta$ defined in (2.9) is necessarily surjective. Applying the second projections $\pi$ and $\bar{\pi}$ of $E(S) \rtimes \Sigma(S)$ and of $(E(S) / \mathcal{D}) \rtimes \Sigma(S / \gamma)$, respectively, we also see that the group homomorphism $\chi$ defined in (2.8) is surjective. Since these projections induce the least group congruences, Diagram 2 can be completed by the isomorphism $\bar{\iota}: \Sigma(S / \gamma) \rightarrow S / \sigma$, so that it be commutative:

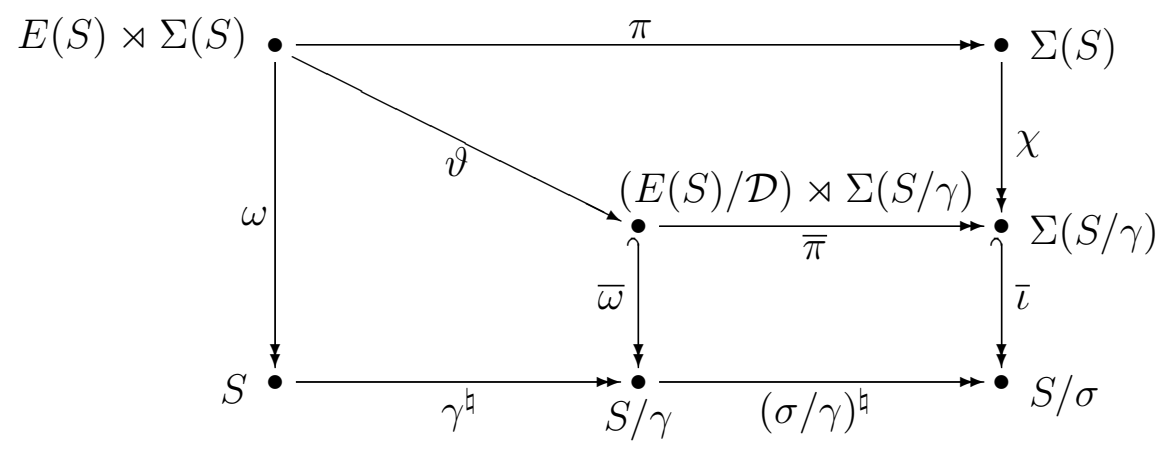

Diagram 2

By a left inverse of $\chi$ we mean a mapping $\varepsilon: \Sigma(S / \gamma) \rightarrow \Sigma(S)$ such that $\varepsilon \chi=\mathrm{id}_{S / \gamma}$. Notice that this equality is equivalent to requiring that the image of $\varepsilon$ forms a transversal for the kernel of $\chi$. If, moreover, 
$\varepsilon$ assigns the identity $\left(\mathrm{id}_{S}, \mathrm{id}_{S}\right) \in \Sigma(S)$ to the identity $\left(\mathrm{id}_{S / \gamma}, \mathrm{id}_{S / \gamma}\right) \in$ $\Sigma(S / \gamma)$ then $\varepsilon$ is termed a unitary left inverse of $\chi$.

Theorem 3.6. Let $S$ be an E-unitary almost factorizable orthodox semigroup. For every unitary left inverse $\varepsilon$ of $\chi$, the mapping $\xi=\varepsilon \beta$ (see (2.5)) satisfies conditions (Q0)-(Q2), and $S$ is isomorphic to the $Q$-product $Q(E(S), \Sigma(S / \gamma) ; \xi)$.

Proof. By the previous argument, the kernel $\kappa$ of $\omega$ is an idempotent separating $E$-unitary congruence on $E(S) \rtimes \Sigma(S)$, and so $\omega$ induces an isomorphism from $(E(S) \rtimes \Sigma(S)) / \kappa$ onto $S$. Moreover, $S / \sigma$ is isomorphic to $\Sigma(S / \gamma)$. Thus, by identifying them via $\bar{\iota}$, we see that the normal subgroup of $\Sigma(S)$ corresponding to $\kappa$ according to Proposition 3.1 is just the kernel of $\chi$. Therefore the proof of the converse part of Theorem 3.5, applied for the transversal determined by $\varepsilon$, immediately implies the assertion.

\section{SEMIDIRECT PRODUCTS OF BANDS BY GROUPS}

In this section we investigate under what conditions a factor semigroup of a semidirect product of a band by a group over an idempotent separating $E$-unitary congruence is isomorphic to a semidirect product of a band by a group. We present an E-unitary almost factorizable orthodox semigroup which is not isomorphic to a semidirect product of a band by a group, and we provide a necessary and sufficient condition for an E-unitary almost factorizable orthodox semigroup to be isomorphic to such a semidirect product.

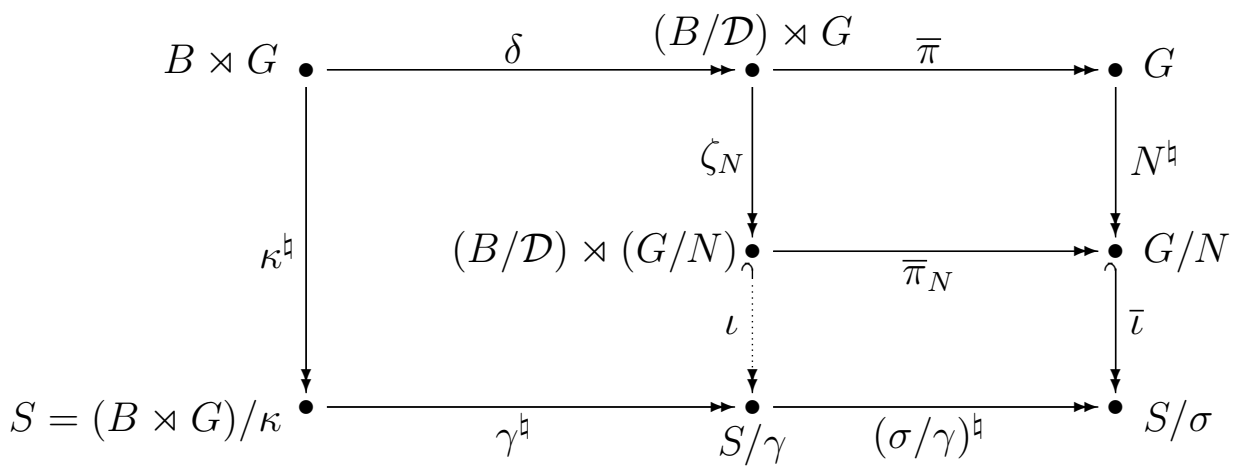

Diagram 3

Now let $B$ be any band and let $G$ be a group acting on $B$. Suppose that $\kappa$ is an idempotent separating $E$-unitary congruence on the semidirect product $B \rtimes G$. For brevity, put $S=(B \rtimes G) / \kappa$. By Proposition 3.1, the relation $\kappa$ is determined by a normal subgroup $N$ of $G$ 
satisfying (C). As it is noticed above, $N$ acts identically on $B / \mathcal{D}$, and we can consider the homomorphisms in Diagram 3 where $\bar{\pi}, \delta$ and $\zeta_{N}$ are defined in $(2.1)-(2.3), \bar{\pi}_{N}$ is the second projection of $(B / \mathcal{D}) \rtimes(G / N)$, and $N^{\natural}$ denotes the natural homomorphism $G \rightarrow G / N, g \mapsto N g$. Furthermore, $\bar{\imath}$ is used to denote the inverse of the isomorphism from $S / \sigma$ onto $G / N$ determined by $\phi$ in Proposition 3.1(2). It is immediate that the diagram consisting of all the arrows of Diagram 3 but $\iota$ is commutative. Since $S$ is $E$-unitary, the congruence $\gamma$ is idempotent pure, and the factor semigroup $S / \gamma$ is an $E$-unitary inverse semigroup. Hence, for any $a, b \in B$ and $g, h \in G$, the elements $(a, g) \kappa$ and $(b, h) \kappa$ of $S$ are $\gamma$-related if and only if they are $\sigma$-related, and

$$
(((a, g) \kappa) \gamma)(((a, g) \kappa) \gamma)^{-1}=(((b, h) \kappa) \gamma)(((b, h) \kappa) \gamma)^{-1}
$$

The former property is equivalent to $N g=N h$ by Proposition 3.1(2). Equation (4.1) is equivalent to $((a, 1) \kappa) \gamma=((b, 1) \kappa) \gamma$, and since $\kappa$ is idempotent separating, it is equivalent to the equality $D_{a}=D_{b}$. Therefore

$$
\iota:(B / \mathcal{D}) \rtimes(G / N) \rightarrow S / \gamma,\left(D_{a}, N g\right) \mapsto((a, g) \kappa) \gamma
$$

is a bijection, and it makes the diagram commutative. By definitions, one can check that $\iota$ is, in fact, an isomorphism.

Now suppose that $S$ is isomorphic to a semidirect product of a band by a group. Then the band is necessarily isomorphic to $E(S)$, and so to $B$, and the group to $S / \sigma$ and so to $G / N$. Thus there is an action of $G / N$ on $B$ and an isomorphism $\psi$ from $S$ onto the respective semidirect product $B \rtimes(G / N)$ such that the following two properties hold. First, $\psi \pi^{\prime}$, where $\pi^{\prime}$ is the second projection of $B \rtimes(G / N)$ is just $\phi$ in Proposition 3.1(2), and second, the restriction of $\psi$ to the bands of idempotents is 'essentially identical', more precisely, we have

$$
((a, 1) \kappa) \psi=(a, N)
$$

for every $a \in B$. The action of $G / N$ on $B$ determines an action of $G / N$ on $B / \mathcal{D}$, and it defines a semidirect product that we denote by $(B / \mathcal{D}) \rtimes^{\prime}(G / N)$ in order to distinguish it from the semidirect product $(B / \mathcal{D}) \rtimes(G / N)$ in Diagram 3 which is introduced in Section 2. Consider the surjective homomorphism (cf. (2.2))

$$
\bar{\delta}: B \rtimes(G / N) \rightarrow(B / \mathcal{D}) \rtimes^{\prime}(G / N),(a, N g) \mapsto\left(D_{a}, N g\right) .
$$

It induces the least inverse semigroup congruence on $B \rtimes(G / N)$, and so $(B / \mathcal{D}) \rtimes(G / N)$ and $(B / \mathcal{D}) \rtimes^{\prime}(G / N)$ are isomorphic. What is more, it is easy to check by taking into account the definition of $\psi$ that $(B / \mathcal{D}) \rtimes(G / N)$ and $(B / \mathcal{D}) \rtimes^{\prime}(G / N)$ coincide. In particular, this means that the two actions of $G / N$ on $B / \mathcal{D}$, namely, the action determined by 
the action of $G / N$ on $B$ and the action defining the semidirect product $(B / \mathcal{D}) \rtimes(G / N)$ coincide. This property can be formulated in the way that, for any $a \in B$ and $g \in G$, we have

$$
{ }^{N g} a \mathcal{D}^{g} a \text {. }
$$

This implies that $B \rtimes(G / N), \psi$ and $\bar{\delta}$ fit in Diagram 3 so that $\psi \bar{\delta} \iota=\gamma^{\natural}$, see Diagram 4.

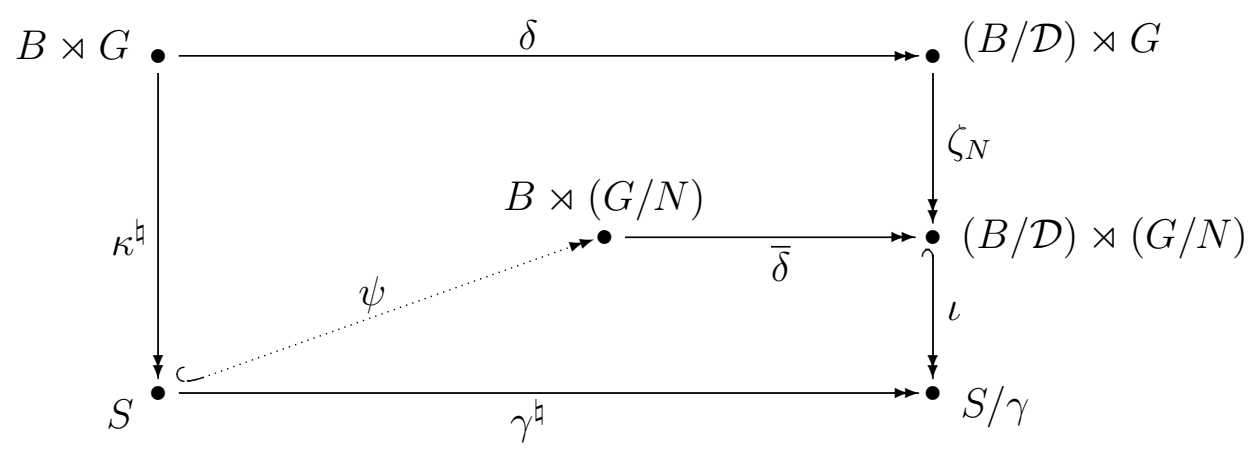

Diagram 4

Let $a, e \in B$ and $g \in G$ such that $((a, g) \kappa) \psi=(e, N g)$. Then we have

$$
(e, N) \mathcal{R}(e, N g)=((a, g) \kappa) \psi \mathcal{R}((a, 1) \kappa) \psi=(a, N)
$$

and

$$
\left({ }^{N g^{-1}} e, N\right) \mathcal{L}(e, N g)=((a, g) \kappa) \psi \mathcal{L}\left(\left(g^{-1} a, 1\right) \kappa\right) \psi=\left(g^{-1} a, N\right)
$$

Hence we obtain that $e \mathcal{R} a$ and ${ }^{N g^{-1}} e \mathcal{L}^{g^{-1}} a$, and so $a \mathcal{R} e \mathcal{L}^{N g}\left(g^{-1} a\right)$ follows. Thus $e=a \cdot{ }^{N g}\left(g^{-1} a\right)$, and this implies

$$
((a, g) \kappa) \psi=\left(a \cdot{ }^{N g}\left(g^{-1} a\right), N g\right)
$$

for every $a \in B$ and $g \in G$. Since $\psi$ is an isomorphism, we have

$$
\begin{aligned}
\left(a b \cdot{ }^{N g}\left(g^{-1}(a b)\right), N g\right) & =((a b, g) \kappa) \psi=((a, 1) \kappa) \psi \cdot((b, g) \kappa) \psi \\
& =(a, N)\left(b \cdot{ }^{N g}\left(g^{-1} b\right), N g\right) \\
& =\left(a b \cdot{ }^{N g}\left(g^{-1} b\right), N g\right)
\end{aligned}
$$

for every $a, b \in B$ and $g \in G$. Thus $a b \cdot{ }^{N g}\left(g^{-1}(a b)\right)=a b \cdot{ }^{N g}\left(g^{-1} b\right)$. Substituting ${ }^{g} a,{ }^{g} b$ for $a, b$, respectively, this implies ${ }^{g}(a b) \cdot{ }^{N g}(a b)={ }^{g}(a b)$. ${ }^{N g} b$ whence we infer ${ }^{N g}(a b) \mathcal{L}^{g}(a b) \cdot{ }^{N g} b$ by $(4.3)$, and so $a b \mathcal{L}^{N g^{-1}}(g(a b)) \cdot b$ follows for every $a, b \in B$ and $g \in G$. Starting with the equality 
$\left(\left(a \cdot{ }^{g} b, g\right) \kappa\right) \psi=((a, g) \kappa) \psi \cdot((b, 1) \kappa) \psi(a, b \in B, g \in G)$, a similar argument provides the equality

$$
\left(a \cdot{ }^{g} b\right) \cdot{ }^{N g}\left(g^{-1}\left(a \cdot{ }^{g} b\right)\right)=a \cdot{ }^{N g}\left(g^{-1} a\right) \cdot{ }^{N g} b .
$$

Substituting ${ }^{g} a$ for $a$, hence we deduce ${ }^{g}(a b) \cdot{ }^{N g}(a b)={ }^{g} a \cdot{ }^{N g}(a b)$, and this implies by (4.3) that ${ }^{g}(a b) \mathcal{R}^{g} a \cdot{ }^{N g}(a b)$, whence $a b \mathcal{R} a \cdot g^{-1}\left({ }^{N g}(a b)\right)$. Thus we have proved the only if part of the following assertion.

Proposition 4.1. Let $B$ be a band, $G$ a group acting on $B$, and let $\kappa$ be an idempotent separating E-unitary congruence on $B \rtimes G$. Denote by $N$ the normal subgroup of $G$ corresponding to $\kappa$ by Proposition 3.1. Then the factor semigroup $(B \rtimes G) / \kappa$ is isomorphic to a semidirect product of a band by a group if and only if there exists an action of $G / N$ on $B$ such that

$$
a b \mathcal{R} a \cdot g^{-1}\left({ }^{N g}(a b)\right) \quad \text { and } \quad a b \mathcal{L}^{N g^{-1}}\left({ }^{g}(a b)\right) \cdot b
$$

for every $a, b \in B$ and $g \in G$.

Proof. Suppose that $G / N$ acts on $B$ such that (4.5) holds. Consider the mapping $\psi: S \rightarrow B \rtimes(G / N)$ defined by the rule (4.4). We verify that $\psi$ is an isomorphism. First of all, observe that (4.5) implies (4.3). For, applying (4.5) in case $b=a$, we deduce that $D_{N g^{-1}\left(g_{a}\right)} \geq D_{a}$ and $D_{g^{-1}\left(N g_{a}\right)} \geq D_{a}$. Hence $D_{g_{a}} \geq D_{N g_{a}}$ and $D_{N g_{a}} \geq D_{g_{a}}$ follow, respectively, which prove (4.3).

The fact that $\psi$ is bijective can be easily checked by applying (4.3) and the properties of $N$ in Proposition 3.1. Indeed, if

$$
\left(a \cdot{ }^{N g}\left(g^{-1} a\right), N g\right)=\left(b \cdot N h\left(h^{-1} b\right), N h\right)
$$

for some $a, b \in B$ and $g, h \in G$, then $N g=N h, a \mathcal{R} b$ and $g^{-1} a \mathcal{L}^{h^{-1}} b$, and they immediately imply together with (4.3) that $a=b$. This establishes injectivity. Furthermore, by (4.3), we can easily see that $\left(\left(b \cdot{ }^{g}\left({ }^{N-1} b\right), g\right) \kappa\right) \psi=(b, N g)$ for any $b \in B$ and $g \in G$ thus proving surjectivity.

Finally, we check that $\psi$ is a homomorphism. Let $a, b \in B$ and $g, h \in$ $G$. In order to show that $((a, g) \kappa \cdot(b, h) \kappa) \psi=((a, g) \kappa) \psi \cdot((b, h) \kappa) \psi$, it suffices to prove that the band components of the left and right hand sides are both $\mathcal{R}$ - and $\mathcal{L}$-related. Again by (4.3), this is equivalent to verifying the relations

$$
a \cdot{ }^{g} b \mathcal{R} a \cdot{ }^{N g}\left({ }^{-1} a\right) \cdot{ }^{N g} b
$$


and

$$
{ }^{N g h}\left((g h)^{-1}\left(a \cdot{ }^{g} b\right)\right) \mathcal{L}^{N g}\left(g^{-1} a\right) \cdot{ }^{N g}\left(b \cdot{ }^{N h}\left(h^{-1} b\right)\right) .
$$

By the first relation in (4.5), we deduce that

$$
\begin{aligned}
a \cdot{ }^{N g}\left({ }^{-1} a\right) \cdot{ }^{N g} b & =a \cdot{ }^{N g}\left({ }^{-1} a \cdot b\right) \\
& \mathcal{R} \quad a \cdot g\left({ }^{N g^{-1}}\left(a \cdot{ }^{N g}\left({ }^{-1} a \cdot b\right)\right)\right) \\
& =a \cdot{ }^{g}\left({ }^{N g^{-1}} a\right) \cdot a \cdot{ }^{g} b=a \cdot{ }^{g} b .
\end{aligned}
$$

In the last step we applied (4.3) to infer $a \cdot{ }^{g}\left({ }^{N g^{-1}} a\right) \cdot a=a$. Thus (4.6) is proved. Similarly, by the second relation in (4.5), we obtain

$$
\begin{aligned}
N g h\left((g h)^{-1}\left(a \cdot{ }^{g} b\right)\right) & =N g h\left((g h)^{-1} a \cdot h^{-1} b\right) \\
& \mathcal{L} \quad N g h\left(N(g h)^{-1}\left(g h\left((g h)^{-1} a \cdot h^{-1} b\right)\right) \cdot h^{-1} b\right) \\
& =a \cdot{ }^{g} b \cdot{ }^{N g h}\left(h^{-1} b\right)
\end{aligned}
$$

and

$$
\begin{aligned}
N g\left(g^{-1} a\right) \cdot N g\left(b \cdot{ }^{N h}\left(h^{-1} b\right)\right) & =N g\left(g^{-1} a \cdot b\right) \cdot{ }^{N g h}\left(h^{-1} b\right) \\
& \left.\mathcal{L}{ }^{N g}\left({ }^{N g^{-1}}\left(g^{g} g^{-1} a \cdot b\right)\right) \cdot b\right) \cdot N g h\left(h^{-1} b\right) \\
& =a \cdot{ }^{g} b \cdot{ }^{N g} b \cdot{ }^{N g h}\left(h^{-1} b\right) \\
& =a \cdot{ }^{g} b \cdot{ }^{N g h}\left(h^{-1} b\right),
\end{aligned}
$$

where in the last step we again utilized (4.3) to see that ${ }^{g} b \cdot{ }^{N g} b$. ${ }^{N g h}\left(h^{-1} b\right)={ }^{g} b \cdot{ }^{N g h}\left(h^{-1} b\right)$. This completes the proof of $(4.7)$.

We have noticed in the previous proof that the conditions in Proposition 4.1 imply property (4.3). Conversely, if the band $B$ is left normal then property (4.3) implies the equalities in the proposition. Therefore we obtain the following corollary.

Corollary 4.2. Let $B$ be a left normal band, $G$ a group acting on $B$, and let $\kappa$ be an idempotent separating E-unitary congruence on $B \rtimes G$. Denote by $N$ the normal subgroup of $G$ corresponding to $\kappa$ by Corollary 3.3. Then the factor semigroup $(B \rtimes G) / \kappa$ is isomorphic to a semidirect product of a band by a group if and only if there exists an action of $G / N$ on $B$ such that (4.3) holds for every $a \in B$ and $g \in G$.

This corollary allows us to provide a finite, E-unitary almost factorizable orthodox semigroup with a left normal band of idempotents which is not isomorphic to a semidirect product of a band by a group. Note that this semigroup is generalized inverse and $\mathcal{R}$-unipotent. Thus, 
among the orthodox semigroups, it is as close to being inverse as possible.

Denote by $Y$ the following semilattice:

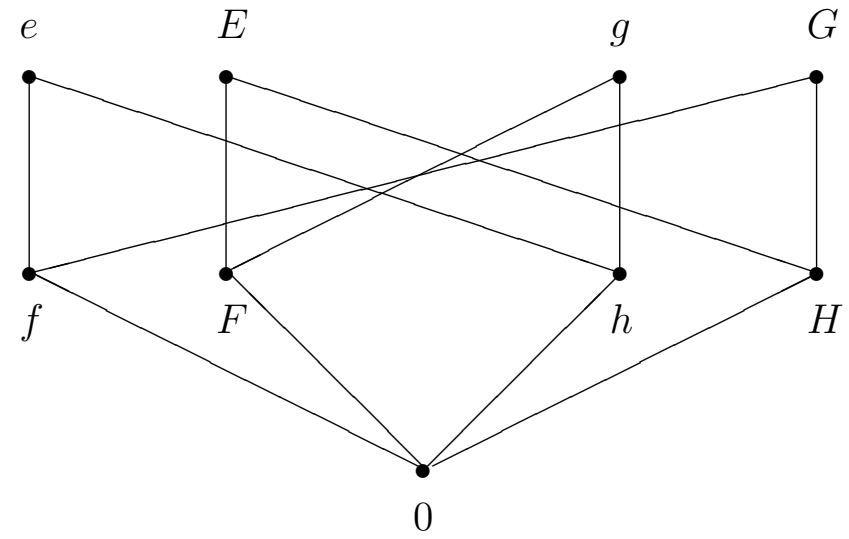

Diagram 5

Define a left normal band $B$ with zero element 0 which is a semilattice $Y$ of left zero semigroups where each non-zero $\mathcal{D}$-class has two elements. As usual, let us identify $B / \mathcal{D}$ with $Y$. For every $x \in$ $\{e, E, f, F, g, G, h, H\}$ we denote the elements of the $\mathcal{D}$-class $x$ by $x_{1}$ and $x_{2}$. Let the natural partial order of $B$ be defined by the relations $x_{i}<y_{i}$ for almost every $i \in\{1,2\}$ and $x, y \in Y$ with $x<y$. The only exceptions are the following two cases: $F_{1}<g_{2}$ instead of $F_{1}<g_{1}$ and, similarly, $F_{2}<g_{1}$ instead of $F_{2}<g_{2}$. This 'twist' is the heart of our construction. It is well known that the natural partial order determines the structure homomorphisms, and so the multiplication of $B$.

An action of the four-element cyclic group $\mathbf{Z}_{4}$ can be defined on $B$ by assigning the permutation

$$
\varsigma=\left(\begin{array}{llll}
e_{1} & E_{1} & e_{2} & E_{2}
\end{array}\right)\left(\begin{array}{llll}
f_{1} & F_{1} & f_{2} & F_{2}
\end{array}\right)\left(h_{1} H_{1} h_{2} H_{2}\right)\left(\begin{array}{llll}
g_{1} & G_{1} & g_{2} & G_{2}
\end{array}\right) \text {. }
$$

of order 4 to $\overline{1}$. In order to verify that $\varsigma$ is, indeed, an automorphism of $B$, it suffices to check that $x<y$ implies $x \varsigma<y \varsigma$ for every $x, y \in B$. Due to the 'twist' in the construction, this property holds. For example $f_{1}<G_{1}$, and indeed $f_{1} \varsigma=F_{1}<g_{2}=G_{1} \varsigma$. Denote by $B \rtimes \mathbf{Z}_{4}$ the semidirect product defined by this action.

Put $N=\{\overline{0}, \overline{2}\}$ which is a 2-element (normal) subgroup of $\mathbf{Z}_{4}$. By Corollary 3.3, $N$ determines an idempotent separating E-unitary congruence $\kappa$ on this semidirect product, and the factor semigroup $S=\left(B \rtimes \mathbf{Z}_{4}\right) / \kappa$ is an $E$-unitary almost factorizable orthodox semigroup with greatest group homomorphic image isomorphic to $\mathbf{Z}_{2}$. 
By Corollary 4.2, $S$ is isomorphic to a semidirect product of a band by a group if and only if there exists an action of the group $\mathbf{Z}_{\mathbf{2}}$ on $B$ such that the automorphism assigned to the generating element $\overline{1}$ of $\mathbf{Z}_{\mathbf{2}}$ determines the following permutation of the $\mathcal{D}$-classes of $B$, that is, of the elements of $Y$ :

$$
(e E)(f F)(g G)(h H) \text {. }
$$

However, observe that the image of $e_{1}$ under the automorphism corresponding to $\overline{1}$ determines the whole automorphism, so, actually there are at most two automorphisms: one maps $e_{1}$ to $E_{1}$, and another maps $e_{1}$ to $E_{2}$. Suppose that $e_{1}$ is mapped to $E_{1}$. In this case, $e_{2}$ is necessarily mapped to $E_{2}$ since the image of $e_{2}$ lies in the $\mathcal{D}$-class $E$. In this case the images of the elements of the $\mathcal{D}$-classes $f, F, h, H$ are determined by the natural partial order: $f_{1}$ is mapped to $F_{1}, h_{1}$ to $H_{1}$, and so on. However, if $f_{1}$ is mapped to $F_{1}$ then $f_{1}<G_{1}$ implies that $G_{1}$ is mapped to $g_{2}$. Similarly, $H_{1}$ is mapped to $h_{1}$, thus $G_{1}$ is necessarily mapped to $g_{1}$, which is a contradiction. Similarly, one can see that the assumption that $e_{1}$ is mapped to $E_{2}$ also leads to a contradiction. Thus $S$ cannot be isomorphic to a semidirect product of a band by a group. So we have proved the following result.

Theorem 4.3. There exists a finite, E-unitary almost factorizable orthodox semigroup with a left normal band of idempotents which is not isomorphic to a semidirect product of a band by a group.

Finally, we turn to giving a necessary and sufficient condition, by means of intrinsic parameters, for an E-unitary almost factorizable orthodox semigroup to be isomorphic to a semidirect product of a band by a group.

Theorem 4.4. For any orthodox semigroup $S$, the following conditions are equivalent:

(1) $S$ is isomorphic to a semidirect product of a band by a group,

(2) $S$ is E-unitary, almost factorizable, and the group extension determined by $\chi$ in (2.8) is splitting.

Proof. Recall that the group extension determined by $\chi$ is splitting if and only if $\chi$ possesses a left inverse which is a homomorphism.

$(2) \Rightarrow(1)$. Let $S$ be an $E$-unitary almost factorizable orthodox semigroup, and suppose that the group extension determined by $\chi$ is splitting. Then there exists a left inverse $\varepsilon$ of $\chi$ which is a homomorphism. In this case $\xi=\varepsilon \beta$ is also a homomorphism, and the $Q$-product $Q(E(S), \Sigma(S / \gamma) ; \xi)$ is a semidirect product. The assertion follows by Theorem 3.6. 
$(1) \Rightarrow(2)$. Assume that $S=B \rtimes H$ where $B$ is a band and $H$ is a group. Then $S$ is an $E$-unitary almost factorizable orthodox semigroup. The congruence induced by the second projection $\pi^{\prime}$ of $S$ is $\sigma$, and so $H$ is isomorphic to $S / \sigma$. The argument before Theorem 3.6 implies that $\bar{\iota}: \Sigma(S / \gamma) \rightarrow S / \sigma,\left(\lambda_{\gamma}, \rho_{\gamma}\right) \mapsto(e \rho) \sigma$, where $(\lambda, \rho) \in \Sigma(S)$ and $e \in$ $E(S)$, is an isomorphism, and so $\bar{\iota}^{\prime}: \Sigma(S / \gamma) \rightarrow H,\left(\lambda_{\gamma}, \rho_{\gamma}\right) \mapsto(e \rho) \pi^{\prime}$, where $(\lambda, \rho) \in \Sigma(S)$ and $e \in E(S)$, is also an isomorphism. Since a semidirect product is a special $Q$-product, we obtain from the proof of the direct part of Theorem 3.5 that, for any $u \in H$, we have $\left(\lambda_{u}, \rho_{u}\right) \in$ $\Sigma(S)$, where $\rho_{u}$ and $\lambda_{u}$ are defined in (3.6) and (3.7), respectively. Since $\eta$ is trivial in our case, we simply have

$$
(a, h) \rho_{u}=(a, h u) \quad \text { and } \quad \lambda_{u}(a, h)=\left({ }^{u} a, u h\right) .
$$

It is routine to check that $\mu: H \rightarrow \Sigma(S), u \mapsto\left(\lambda_{u}, \rho_{u}\right)$, and, consequently, $\varepsilon=\bar{\iota}^{\prime} \mu$, are homomorphisms. Moreover, by the definition of $\bar{\iota}^{\prime}$ and $\mu$, we have

$$
\left(\left(\lambda_{u}\right)_{\gamma},\left(\rho_{u}\right)_{\gamma}\right) \varepsilon=\left(\left(\left(\lambda_{u}\right)_{\gamma},\left(\rho_{u}\right)_{\gamma}\right) \bar{\iota}^{\prime}\right) \mu=u \mu=\left(\lambda_{u}, \rho_{u}\right)
$$

therefore $\varepsilon$ is a unitary left inverse of $\chi$. Thus the group extension corresponding to $\chi$ is, indeed, splitting.

\section{REFERENCES}

[1] M. Hartmann, Almost factorizable orthodox semigroups Semigroup Forum $\mathbf{7 4}$ (2007), 106-124.

[2] J. M. Howie, Fundamentals of Semigroup Theory, London Math. Soc. Monographs, New series 12, Clarendon Press, New York-Oxford, 1995.

[3] M. V. Lawson, Inverse semigroups: The Theory of Partial Symmetries, World Scientific, Singapore, 1998.

[4] M. Petrich, Inverse semigroups, Wiley \& Sons, New York, 1984.

Bolyai Institute, University of Szeged, Aradi vértanúk tere 1, H6720 Szeged, Hungary

E-mail address: hartm@math.u-szeged.hu

Bolyai Institute, University of Szeged, Aradi vértanúk tere 1, H6720 Szeged, Hungary

E-mail address: m.szendrei@math.u-szeged.hu 